\title{
Roles of p38 MAPK in the regulation of the inflammatory response to swine influenza virus-induced acute lung injury in mice
}

\author{
D. WEI, Z.-H. HUANG, R.-H. ZHANG, C.-L. WANG, M.-J. XU, B.-J. LIU, G.-H. WANG, T. XU*
}

Department of Veterinary Medicine, College of Animal Science, HeBei North University, Zhangjiakou, 075131, Hebei, P. R. China

Received November 25, 2013; accepted November 19, 2014

\begin{abstract}
Summary. - Swine influenza virus (SIV), one of the most important zoonotic agents, is associated with major public health concerns. The current study was conducted to investigate the role of p 38 mitogen-activated protein kinase (p38 MAPK) in the regulation of the inflammatory response to acute lung injury (ALI) induced by SIV of H9N2 subtype (H9N2-SIV) in mice. For this purpose, BALB/c mice were intranasally infected with $20 \mathrm{LD}_{50}$ of H9N2-SIV (infected group), while non-infected mice served as control (control group). To assess the effect of p38 MAPK, its specific inhibitor SB203580 was employed followed by SIV infection (SB group). At various times after infection, mouse lungs were subjected to pathological and histological observations and detection of inflammatory cytokines tumor necrosis factor a (TNF)- $\alpha$, interleukin (IL)-1 $\beta$, IL-6, and IL-10 and phosphorylated p38 MAPK. The obtained results showed obvious inflammatory responses, injury and raised levels of inflammatory cytokines and phosphorylated p38 MAPK in the lungs of virus-infected mice. In the mice inoculated with the virus alone, the level of phosphorylated p38 MAPK increased from day 2 and peaked at day 6 post infection (p.i.). However, SB203580 caused lower increases in inflammatory cytokines and phosphorylated p38 MAPK and a milder lung injury. These findings indicate that the activation of p38 MAPK upregulated the inflammatory responses to H9N2-SIV-induced ALI, increased its severity and promoted the production of inflammatory cytokines.
\end{abstract}

Keywords: p38 MAPK; swine influenza virus; inflammatory response; acute lung injury; SB203580; mouse

\section{Introduction}

Acute lung injury (ALI) and its more severe form, acute respiratory distress syndrome (ARDS), are characterized by overwhelming lung inflammation and increased $\mathrm{mi}-$ crovascular permeability, causing diffuse lung edema and mechanical dysfunction (Wheeler and Bernard, 2007). ALI is the primary set of symptoms of influenza virus infection

*Corresponding author. E-mail: hbzjkwd@163.com; xutong1969@ sohu.com; phone: +86-18931318516.

Abbreviations: ALI = acute lung injury; IL = interleukin; MAPK = mitogen-activated protein kinase; p.i. = post infection; i.p. = intraperitoneally; SB group = SB203580; SIV = swine influenza virus; TNF- $\alpha=$ tumor necrosis factor $\alpha$; $/ \mathrm{D}=$ wet $/$ dry weight ratios in mammals, but exact pathogenesis of this virus remains uncertain. In recent years, studies on the pathogenesis of influenza viruses have mainly focused on inflammatory cytokines and mediators. Swine influenza caused by swine influenza virus (SIV) is an acute and highly contagious respiratory disease. Pigs are the most common hosts of human influenza viruses, avian influenza viruses, and SIV, and they play a "mixer" role during co-infection by human and avian influenza viruses. SIV infected pigs are often without any clinical signs. Virus recombination which occurs in pigs, leads to new strains and increases the prevalence of influenza virus infection. In addition research demonstrated that humans can also be infected with SIV (Newman et al., 2008; Adiego Sancho et al., 2009; Van Reeth et al., 2009). Therefore, research of swine influenza is of great importance for protecting the public health. 
Signaling through mitogen-activated protein kinase (MAPK), a serine/threonine protein kinase, is one of the four major signaling pathways of eukaryotic cells (Herlaar et al., 1999). p38 MAPK was the third MAPK to be identified. p38 MAPK plays an important role in regulating cell proliferation, apoptosis, inflammation, stress, cytokine production, gene transcription, and cytoskeleton recognition. Previous investigations proved that p38 MAPK and inflammation were closely related and that $\mathrm{p} 38 \mathrm{MAPK}$ could regulate the expression of inflammatory cytokines in vitro (Huang et al., 2004; SchnyderCandrian et al., 2005). Powell et al. (2004) confirmed that the p38 MAPK signaling pathway played an essential role in the regulation of lung injury. However, the influence of p 38 MAPK on inflammation associated with ALI induced by SIV subtype H9N2 (H9N2-SIV) has not been reported previously. SB203580 is one of the most commonly used p38 MAPK inhibitors, and it inhibits p38 MAPK phosphorylation without affecting ERK1/2, JNK, and ERK5 (Ono et al., 2000; Peifer et al., 2006). Previous studies suggested that through the inhibition of p38 MAPK activation and expression, SB203580 effectively inhibited the expression of inflammatory cytokines such as TNF- $\alpha$ and IL- $1 \beta$, reduced the permeability of pulmonary capillary, and prevented pathological damage in the lungs.

In this study, BALB/c mice with ALI induced by H9N2SIV infection were used as animal model. Pathological changes, levels of inflammatory cytokines and phosphorylated p38 MAPK in mouse lungs were investigated during the ALI progression. To assess the effect of p38 MAPK, its specific inhibitor SB203580 was employed. The obtained results demonstrated that 338 MAPK was involved in the regulation of the inflammatory responses to ALI induced by H9N2-SIV infection in mouse lungs.

\section{Materials and Methods}

Virus. A/swine/HeBei/012/2008(H9N2) virus (H9N2-SIV) was inoculated into 10-day-old SPF chicken embryos (Beijing Laboratory Animal Research Center, China) and was consecutively blind passaged for 3 generations. The allantoic fluid was collected and stored at $-70^{\circ} \mathrm{C}$.

Experiment design. One hundred and eighty 6-8-week-old SPF female BALB/c mice (Beijing HFK Bioscience Co. Ltd, China) were divided into 3 groups of 60 mice each. BALB/c mice were intranasally infected with H9N2-SIV $\left(0.1 \mathrm{ml}\right.$, containing $\left.20 \mathrm{LD}_{50}\right)$ (infected group), and control mice (control group) by the same dose of noninfectious allantoic fluid, which had been diluted 1:5 by sterile saline buffer. To assess the effect of p38 MAPK, its specific inhibitor SB203580 was employed. First, $1.0 \mathrm{mg}$ of SB203580 was dissolved in $100 \mu \mathrm{l}$ of DMSO and then it was diluted with sterile saline buffer to obtain a final concentration of $1.0 \mathrm{mg} / \mathrm{ml}$. Finally, the mice were intraperitoneally (i.p.) injected with a dose of $5 \mathrm{mg}$ SB203580 per kg/mouse (Fujise et al., 2006; Widmann et al., 1999) and after 1 hour they were infected with H9N2-SIV as aforementioned (SB group). Detailed methods of model making can be found in the literature (Dong et al., 2012). The inoculated mice were separated and kept in the bio-safety level 3+ laboratories with access to water and food ad libitum. The study was approved by the Animal Care Committee of Hebei North University (No. 2012-1-0-08, China).

Pathology of lungs. Five mice in each group were weighed and sacrificed on days $2,4,6,8$, and 14 p.i., and the whole wet lungs were weighed to assess lung coefficient. The W/D ratios were determined by weighing the right lung before and after oven desiccation at $80^{\circ} \mathrm{C}$. $\mathrm{W} / \mathrm{D}$ ratio was used as an indicator of lung edema (Majeski et al., 2003). Left lobes of lungs were fixed in $4 \%$ paraformaldehyde and embedded in paraffin. The samples for microscopic examination were stained with hematoxylin and eosin.

Assay of inflammatory cytokines. Pulmonary homogenate was centrifuged at $3,000 \mathrm{x} \mathrm{g}$ for $15 \mathrm{~min}$ at $4^{\circ} \mathrm{C}$, the supernatant was collected and used to determine the cytokines. The expression of TNF- $\alpha$, IL- $1 \beta$, IL- 6 , and IL-10 were determined using a sandwich ELISA kits following the instructions recommended by the manufacturer. The absorbance of the microplate was read at $450 \mathrm{~nm}$.

Western blot analysis. The protein expression of p38 MAPK was measured using a Western blot analysis kit (Boster Biological Technology., LTD, China) according to the manufacturer's protocols. Briefly, frozen lung samples were defrosted and were homogenized with lysis buffer (RIPA with protease inhibitor, with a ratio of lung tissue/ lysis buffer (ml) equal to $1 / 10$ ). The homogenate was sonicated and centrifuged at $10,000 \times \mathrm{g}$ for $10 \mathrm{~min}$ at $4^{\circ} \mathrm{C}$. Then the supernatant was collected and its protein concentrations were determined by BCA protein assay kit (Pierce). The proteins were separated on 10\% SDSpolyacrylamide gel and transferred onto the PVDF membrane. After blocking the nonspecific sites with blocking solution (5\% nonfat dry milk), the blotted membrane was incubated overnight with specific primary antibody against $\beta$-actin and phospho-p38 MAPK (Santa Cruz) at $4^{\circ} \mathrm{C}$. Subsequently, the blotted membrane was washed with PBS-T followed by incubation with the secondary antibody conjugated with horseradish peroxidase (HRP) for $1 \mathrm{hr}$ at room temperature. Blots were again washed with PBS-T and then developed with the DAB Western blot analysis detection system. Blots were visualized using a SmartGel system and Quantity One analysis software (Bio-Rad), and the band density was quantified using Lane $1 \mathrm{D}$ software.

Statistical analysis. All data were expressed as means \pm SD. Statistical analysis was performed with the SPSS statistical software package for Windows, version 13.0 (SPSS, Inc., USA). Differences between groups were examined for statistical significance by two-tailed Student $t$ test. The p-values less than 0.05 were considered statistically significant.

\section{Results}

\section{Pathology of lungs}

Lungs of the control mice were rose pink and soft, and the lobes of the lungs were smooth and moist with no con- 


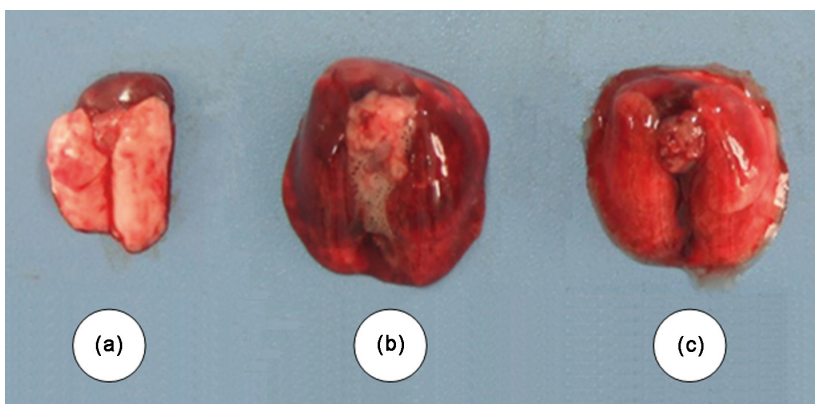

Fig. 1

Pathology of mouse lungs

The legend: Control (a), infected (b) and SB + infected (c) mice.

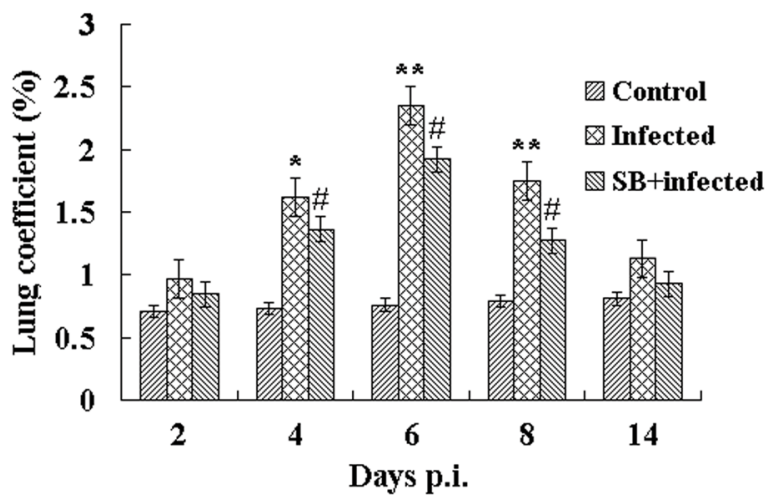

Fig. 2

Changes in the lung coefficient of mice The legend: ${ }^{*} \mathrm{P}<0.05$ vs. control, ${ }^{* *} \mathrm{P}<0.01$ vs. control, ${ }^{*} \mathrm{P}<0.05$ vs. infected.

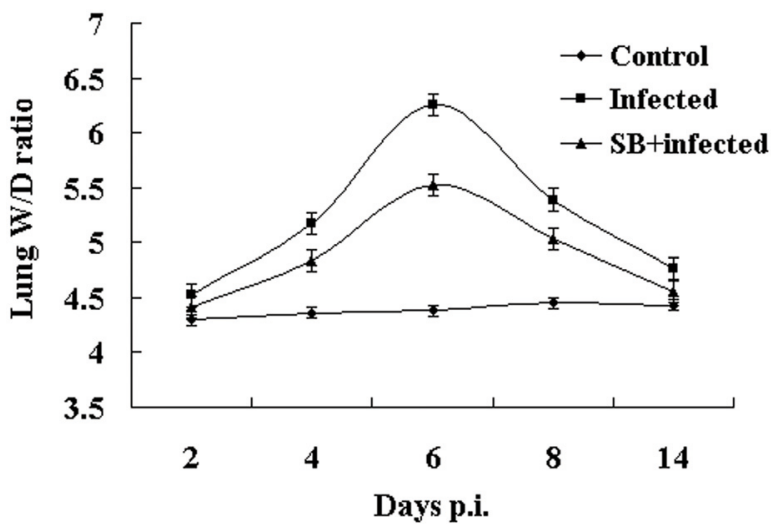

Fig. 3

Changes in the lung W/D of mice solidation regions. Lungs of the infected mice were highly edematous, with profuse areas of hemorrhage and congestion. Furthermore, a region of red hepatization was visible, and bloody foam fluid flowed from the bronchus. Lesions of lungs such as congestion and hemorrhage were less severe in the SB mice as in the infected mice (Fig. 1).

Compared to the control mice, the lung wet weight of infected mice increased slightly on day 2 p.i., but there was no statistical significance in the lung coefficient and W/D ratio. However, the lung coefficient and W/D ratio of infected mice increased significantly $(\mathrm{P}<0.01)$ on day $4-6$ p.i. On day 8 p.i., the lung coefficient and W/D ratio of the infected mice began to recover progressively, but they still had significant difference $(\mathrm{P}<0.01)$. On day 14 p.i., there was no significant difference in the lung coefficient and $\mathrm{W} / \mathrm{D}$ ratio $(\mathrm{P}>0.05)$, and they were close to control data. Additionally, compared to the infected mice, there was no significant difference in the lung coefficient and $W / D$ ratio in the SB mice. On day 14 p.i., the lung coefficient and W/D ratio were similar to the control data (shown in Fig. 2-3).

\section{Histopathology of the lungs}

Lungs of the control mice had no apparent histopathological lesions, alveolus was clear, and alveolar walls were thin.

Compared to the control mice, lungs of the infected mice had apparent histopathological lesions. On day 2 p.i., lung histopathology was characterized by interstitial edema around the small blood vessels and bronchiole with inflammatory cell exudates, and edema fluid were observed in the alveolar lumen (Fig. 4a). On day 4 p.i., alveolar walls were thickened, pulmonary interstitium broadened, and obvious edema was present in interstitium around vessels and bronchiole, and parts of bronchial epithelium were necrotic and sloughed. Moreover, plenty of erythrocytes appeared in alveolar lumen and interstitium (Fig. 4b). On day 6 p.i., plenty of inflammatory cells were exuded and bronchopneumonia developed (Fig. 4c). On day 8 p.i., the histopathological lesions of the infected mice began to recover progressively. Compared to the infected mice, lungs of the SB mice had slightly less apparent histopathological lesions (Fig. 4d,e,f).

\section{Inflammatory cytokines in lungs}

As shown in Table 1, compared with the control mice, the concentrations of TNF- $\alpha$, IL- $1 \beta$, IL- 6 , and IL-10 in lung homogenates were significantly higher in the infected mice $(\mathrm{P}<0.05$ or $\mathrm{P}<0.01)$.

On day 2 p.i., the levels of TNF- $\alpha$ and IL- $1 \beta$ increased rapidly, and peaked at day 4 . On day 6 p.i., the levels started to decline. On day 14 p.i., the levels of TNF- $\alpha$ and IL- $1 \beta$ were reaching control mice. 
Table 1 Inflammatory cytokine levels in mouse lungs

\begin{tabular}{|c|c|c|c|c|c|}
\hline Mice & Days p.i. & TNF- $\alpha$ & IL- $1 \beta$ & IL-6 & IL-10 \\
\hline Control & & $25.76 \pm 6.52$ & $28.28 \pm 5.28$ & $19.35 \pm 8.17$ & $80.57 \pm 12.25$ \\
\hline Infected & \multirow{2}{*}{$2 d$} & $40.31 \pm 8.31^{\diamond}$ & $42.81 \pm 8.63^{\diamond}$ & $23.26 \pm 10.23^{\diamond}$ & $103.73 \pm 18.52^{\Delta}$ \\
\hline SB + infected & & $33.81 \pm 7.26^{\bullet}$ & $38.15 \pm 6.97^{\bullet}$ & $20.67 \pm 10.18^{\diamond}$ & $96.57 \pm 10.53^{\Delta \Delta}$ \\
\hline Infected & \multirow{2}{*}{$4 \mathrm{~d}$} & $44.70 \pm 13.62^{\Delta}$ & $44.21 \pm 12.14^{\Delta}$ & $24.81 \pm 10.72^{\Delta}$ & $141.73 \pm 20.15^{\Delta}$ \\
\hline SB + infected & & $38.70 \pm 12.51^{\Delta \Lambda}$ & $41.93 \pm 8.31^{\Delta \Lambda}$ & $22.22 \pm 9.85^{\diamond}$ & $129.07 \pm 13.76^{\Delta \Lambda}$ \\
\hline Infected & \multirow{2}{*}{$6 \mathrm{~d}$} & $41.81 \pm 9.17^{\Delta}$ & $43.59 \pm 11.92^{\Delta}$ & $33.26 \pm 11.82^{\Delta}$ & $145.73 \pm 15.71^{\diamond}$ \\
\hline SB + infected & & $35.48 \pm 10.68^{\Delta \Delta}$ & $39.05 \pm 12.35^{\Delta \Delta}$ & $29.81 \pm 10.92^{\Delta}$ & $134.23 \pm 20.13^{\diamond}$ \\
\hline Infected & \multirow{2}{*}{$8 \mathrm{~d}$} & $36.26 \pm 5.16^{\diamond}$ & $36.65 \pm 5.87^{\diamond}$ & $31.85 \pm 8.21^{\Delta}$ & $129.90 \pm 11.23^{\diamond}$ \\
\hline SB + infected & & $31.37 \pm 4.51^{\diamond}$ & $32.18 \pm 6.72^{\diamond}$ & $27.54 \pm 5.43^{\Delta 凶}$ & $112.73 \pm 10.56^{\diamond}$ \\
\hline Infected & \multirow{2}{*}{$14 \mathrm{~d}$} & $30.31 \pm 6.07^{\diamond}$ & $31.53 \pm 4.11^{\diamond}$ & $25.81 \pm 7.87^{\diamond}$ & $105.07 \pm 11.87^{\Delta}$ \\
\hline SB + infected & & $28.03 \pm 4.42^{\star}$ & $29.12 \pm 5.37^{\star}$ & $22.85 \pm 6.39^{\bullet}$ & $90.90 \pm 12.33^{\star}$ \\
\hline
\end{tabular}

Note: ${ }^{\diamond} \mathrm{P}<0.05$ vs. control, ${ }^{\Delta} \mathrm{P}<0.01$ vs. control, ${ }^{\bullet} \mathrm{P}<0.05$ vs. infected, ${ }^{\wedge} \mathrm{P}<0.01$ vs. infected.

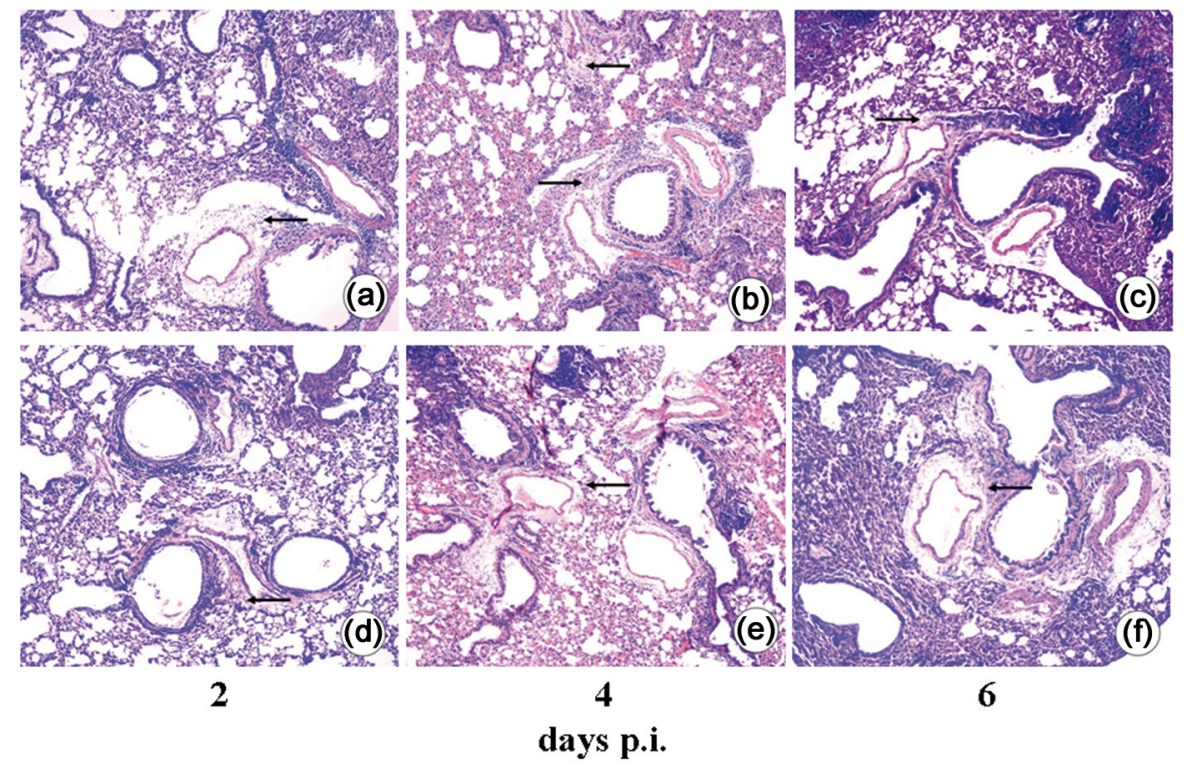

Fig. 4

Histopathology of mouse lungs

The legend: Infected mice (a, b, c), SB + infected mice (d, e, f). Magnification 100x.
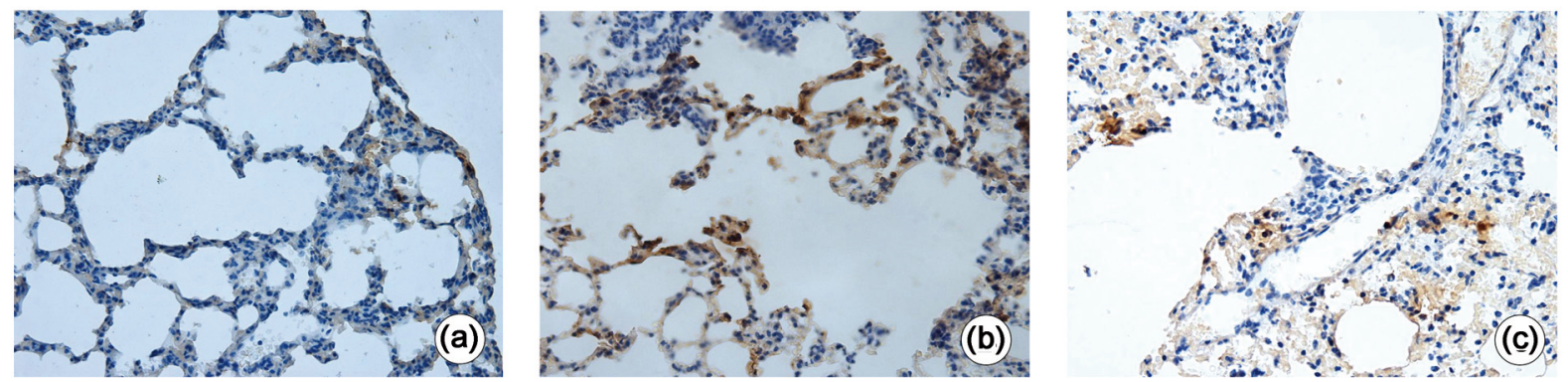

Fig. 5

Levels of phosphorylated p38 MAPK in mouse lungs 6 days p.i.

The legend: Control (a), infected (b) and SB + infected (c) mice. Magnification 400x. 


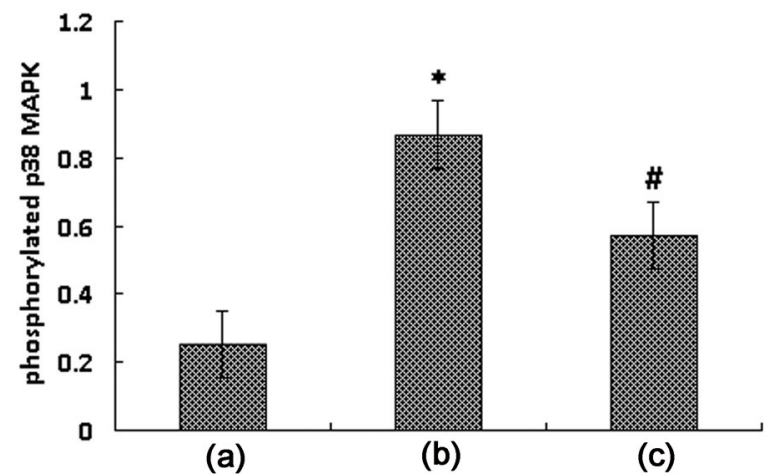

Fig.6

Levels of phosphorylated p38 MAPK in mouse lungs 6 days p.i. The legend: Western blot analysis, control (a), infected (b) and SB + infected (c) mice. ${ }^{*} \mathrm{P}<0.05$ and ${ }^{*} \mathrm{P}<0.05$ vs. control.

On day 2 p.i., the concentrations of IL- 6 and IL-10 were also elevated, and peaked at day 6 . On day 8 p.i., the levels of the two cytokines began to decline slowly. On day 14 p.i., they still remained higher than in control mice.

SB203580 was able to efficiently reduced the production of TNF- $\alpha$, IL-1 $\beta$, IL- 6 , and IL-10 $(\mathrm{P}<0.05$ or $\mathrm{P}<0.01)$.

\section{p38 MAPK in lungs}

Expression of phosphorylated p38 MAPK was determined by immunohistochemistry. The pulmonary tissue of the control mice was normal, and only small amounts of phosphorylated p38 MAPK were found in the epithelial cells of the alveolus and lung airway (Fig. 5a). Expression of phosphorylated p38 MAPK was obviously increased in the infected mice, especially the epithelial cells of the alveolar septum, and myoid cells in the nodular enlargement region of the alveolar ducts also displayed a positive reaction. At the same time, phosphorylated p38 MAPK was widely distributed in the epithelial cells of the lung airway, vascular endothelial cell, cytoplasm, and nucleus. High expression of phosphorylated p38 MAPK was also noted in inflammatory cells (Fig. 5b). Compared to the infected mice, the expression of phosphorylated p38 MAPK in the SB mice has decreased significantly. Only the cytoplasm of epithelial cells of the alveolar septum had a small positive expression of phosphorylated p38 MAPK and expression of capillary endothelial cells in the alveolar septum has disappeared. On day 14 p.i., expression of phosphorylated p38 MAPK was recovered to normal levels (Fig. 5c).

The expression of phosphorylated p38 MAPK protein was determined by Western blot analysis too. The results showed that H9N2-SIV could significantly increase the phosphorylation of p38 MAPK. However, pretreatment with SB203580 could inhibit the phosphorylation of p38 MAPK (Fig. 6).
In all, the results showed that SB203580 could inhibit p38 MAPK signaling pathway efficiently in H9N2-SIV induced ALI of mice

\section{Discussion}

Protein p38 MAPK is an important member of mitogenactivated protein kinase (MAPK) family. It occupies a central role in the regulation of inflammation, and its persistent activation causes the production of the inflammatory cytokines, leading to cascade of effects (Peifer et al., 2006). Following the stimulation of various pathogenic factors of ALI/ARDS, p38 MAPK can be activated. Nick et al. (2000) suggested that the p38 MAPK pathway plays an essential role in the development of ALI. Therefore, study of the relationship between the activation of p38 MAPK and the production of inflammatory cytokines in ALI, will help to elucidate the pathogenesis of ALI/ARDS.

Previous reports supported that the p38 MAPK signaling pathway was involved in many important physiological processes. Blockade of p38 MAPK expression could regulate the inflammatory response, but it also affected normal physiological reactions, a process called p38-mechanismbased toxicity. Therefore, selecting the correct dose of p38 MAPK inhibitors for the analysis was extremely important. The related reports in the literature demonstrated that low doses of SB203580 could effectively inhibit the expression of p38 MAPK without affecting the activation of JNK and ERK (Fujise et al., 2006; Widmann et al., 1999). Therefore, in this study, a dose of $5 \mathrm{mg} / \mathrm{kg}$ /body weight of SB203580 was chosen, and mice were i.p. injected 30-60 mins before the surgery.

The present study suggested that within 14 days p.i., the typical symptoms of ALI were observed, and the activation of $\mathrm{p} 38$ MAPK participated in the occurrence of ALI in the infected and SB mice. However, compared with the infected mice, the severity of lung lesions such as congestion, hemorrhage, edema and the expression of inflammatory cytokines and phosphorylated p38 MAPK were significantly reduced in the SB mice. These results indicated that p38 MAPK was involved in the inflammatory responses to ALI induced by H9N2-SIV. Additionally, the expression of TNF- $\alpha$, IL-1 $\beta$, and IL-6 increased rapidly, resulting in inflammation and organ damage in the infected and SB mice. IL- 6 displayed the same ability as the anti-inflammatory cytokine IL-10, to produce inflammatory responses, suggesting that IL-6 might have promoted both inflammatory and anti-inflammatory functions in the experiment, but the mechanism requires additional research for elucidation. Compared to the changes in TNF- $\alpha$ and IL- $1 \beta$ levels, IL-10 exerted anti-inflammatory effects, and its expression increased rapidly and persistently over time, indicating that IL-10 exerted its effects downstream of TNF- $\alpha$ and IL-1 $\beta$. This finding indicated that 
IL-10 was involved in regulating the body's balance and the recovery of immune function, thereby reducing the inflammatory reaction.

In conclusion, this study demonstrated that the expression of phosphorylated p38 MAPK was increased in H9N2-SIV induced ALI in mice. After treatment with the p38 MAPKspecific inhibitor SB203580, the expression of phosphorylated p38 MAPK was heavily inhibited, and the pathological severity of ALI was lessened. These findings indicated that p38 MAPK activation was involved in regulating inflammatory responses in the lung tissue during ALI induced by H9N2-SIV, promoting the production of inflammatory cytokines, and increasing the severity of ALI.

Acknowledgement. This work was supported by the grants No. C2009001028 and C2011405002 from the Natural Science Foundation of HeBei province of China, and the grants No.ZD20131045 from Key project of HeBei education department.

\section{References}

Adiego Sancho B, Omenaca Teres M, Martinez Cuenca S, Rodrigo Val P, Sanchez Villanueva P, Casas I, Pozo F, Perez Brena P (2009): Human case of swine influenza A(H1N1), Aragon, Spain, November 2008. Euro Surveill. 14, 19120.

Dong W, Li-Feng X, Cun-Lian W, Ming-Ju X, Rui-Hua Z, Ying L, Tong X (2012): A mouse model of swine influenza virus H9N2 infection with acute lung injury. Acta Virol. 56, 227-233. http://dx.doi.org/10.4149/av 201203227

Fujise T, Iwakiri R,Wu B, Amemori S, Kakimoto T, Yokoyama F, Sakata Y, Tsunada S,Fujimoto K (2006): Apoptotic pathway in the rat small intestinal mucosa is different between fasting and ischemia-reperfusion. Am. J. Physiol. Gastrointest. Liver Physiol. 291, G110-116. http://dx.doi. org/10.1152/ajpgi.00393.2005

Herlaar E, Brown Z (1999): p38 MAPK signaling cascades in inflammatory disease. Mol. Med. Today 5, 439-447. http:// dx.doi.org/10.1016/S1357-4310(99)01544-0

Huang C, Jacobson K, Schaller MD (2004): MAP kinase and cell migration. J. Cell Sci.117, 4619-4628. http://dx.doi. org/10.1242/jcs.01481
Majeski EI, Paintlia MK, Lopez AD, Harley RA, London SD, London L (2003): Respiratory reovirus $1 / \mathrm{L}$ induction of intraluminal fibrosis, a model of bronchiolitis oblitreans organizing pneumonia, is dependent on T lymphocytes. Am. J. Pathol. 163, 1467-1479. http://dx.doi.org/10.1016/ $\underline{\text { S0002-9440(10)63504-3 }}$

Newman AP, Reisdorf E, Beinemann J, Uyeki TM, Balish A, Shu B, Lindstrom S, Achenbach J, Smith C, Davis JP (2008). Human case of swine influenza A (H1N1) triple reassortant virus infection, Wisconsin. Emerg. Infect. Dis.14, 1470-1472. http://dx.doi.org/10.3201/eid1409.080305

Nick JA, Young SK, Brown KK, Avdi NJ, Arndt PG, Suratt BT, Janes MS, Henson PM, Worthen GS (2000): Role of p38 mitogen-activated protein kinase in a murine model of pulmonary inflammation. J. Immunol.164, 2151-2159. http://dx.doi.org/10.4049/jimmunol.164.4.2151

Ono K, Han J (2000): The p38 signal transduction pathway: activation and function. Cell Signal. 12, 1-13. http://dx.doi. org/10.1016/S0898-6568(99)00071-6

Peifer C, Wagner G, Laufer S (2006): New approaches to the treatment of in flammatory disorders small molecule inhibitors of p38 MAP kinase. Curr. Top. Med. Chem. 6, 113-149. http://dx.doi.org/10.2174/156802606775270323

Powell CS, Wright MM, Jackson RM (2004): p38mapk and MEK1/2 inhibition contribute to cellular oxidant injury after hypoxia. Am. J. Physiol. Lung Cell. Mol. Physiol. 286, L826833. http://dx.doi.org/10.1152/ajplung.00119.2003

Schnyder-Candrian S, Quesniaux VF, Di Padova F, Maillet I, Noulin N, Couillin I, Moser R, Erard F, Vargaftig BB, Ryffel B, Schnyder B (2005): Dual effects of p38 MAPK on TNF-dependent bronchoconstriction and TNFindependent neutrophil recruitment in lipopolysaccharide-induced acute respiratory distress syndrome. J. Immunol. 175, 262-269. http://dx.doi.org/10.4049/ jimmunol.175.1.262

Van Reeth K, Nicoll A (2009): A human case of swine influenza virus infection in Europe-implications for human health and research. Euro Surveill. 14, 19124.

Wheeler AP and Bernard GR (2007): Acute lung injury and the acute respiratory distress syndrome: a clinical review. Lancet 369, 1553-1564. http://dx.doi.org/10.1016/S01406736(07)60604-7

Widmann C, Gibson S, Jarpe MB, Johnson GL (1999): Mitogen-activated protein kinase: eonservation of a three-kinase module from yeast to human. Physiol. Rev. 79, 143-180. 